\title{
1 Title: Hippocampal place cells as a cryptographic tool that enables 2 animals to secretly cache and retrieve their food
}

4 Authors:

5 Oren Forkosh ${ }^{1,2}$

\section{Affiliations:}

81 Department of Cognitive and Brain Sciences, The Hebrew University of Jerusalem, 9 Jerusalem, Israel

$10{ }^{2}$ Department of Animal Sciences, The Hebrew University of Jerusalem, Rehovot, Israel

\section{Abstract}

13 For animals, the ability to hide and retrieve valuable information, such as the location of 14 food, can mean the difference between life and death. Here, we propose that to achieve 15 this, their brain uses spatial cells similarly to how we utilize encryption for data security. 16 Some animals are able to cache hundreds of thousands of food items annually by each 17 individual and later retrieve most of what they themselves stashed. Rather than memorizing 18 their cache locations as previously suggested, we propose that they use a single 19 cryptographic-like mechanism during both caching and retrieval. The model we developed 20 is based on hippocampal spatial cells, which respond to an animal's positional attention, 21 such as when the animal enters a specific region (place-cells) or gazes at a particular 22 location (spatial-view-cells). We know that the region that activates each spatial cell remains 23 consistent across subsequent visits to the same area but not between areas. This 24 remapping, combined with the uniqueness of cognitive maps, produces a persistent crypto25 hash function for both food caching and retrieval. We also show that the model stores 26 temporal information that helps animals in food caching order preference as previously 27 observed. This behavior, which we refer to as crypto-taxis, might also explain consistent 28 differences in decision-making when animals are faced with a large number of alternatives 29 such as in foraging. 
Main Text

Animals have much to hide. Some species evade potential predators or prey by finding cover or by using camouflage (1), mimicry, and other means of disguise (2). Others conceal their eggs or offspring, mask an illness or an injury to avoid being targeted by predators (3), or stash valuable resources, such as food.

Scatter hoarding is probably the largest-scale manifestation of secretive behavior in the animal kingdom. Many species of animals engage in this behavior, which involves storing food at multiple cache sites to preserve it for times when food is scarce (4). Several bird species, such as the Siberian tit (Poecile cinctus), were observed to cache over 500,000 items per individual in one year (4). While much of the research on scatter hoarding was conducted on birds, this behavior is not specific to them (4): squirrels (5), chipmunks (6), and even foxes $(7,8)$ stash food for times of need. As caching sites cannot be defended, the success of this strategy is often contingent on an animal's ability to keep the stashes away from prying eyes and hard to find (5).

Once the valuables are stashed, scatter hoarders are faced with the considerable challenge of retrieving the hidden items. It was shown that hoarding birds do not randomly seek out cached food (9); rather, they mainly retrieve that which they had themselves hidden - a remarkable feat, especially given the large number of cache sites (10). In the 1950s, Olof Swanberg observed that almost $90 \%$ of Eurasian nutcracker (Nucifraga caryocatactes) excavation holes contained cracked nutshells, indicating successful retrievals (4). In one elegant experiment, Stevens and Krab attached tiny magnets to the legs of marsh tits (Poecile palustris) (12). The birds were then offered serially numbered peanuts labeled with a radioactive isotope, enabling the researchers to locate each bird's caching sites using a scintillation counter (13). Magnet detectors were placed at each location to detect the presence of marked birds. During the experiment, the birds retrieved about $25 \%$ of their own stored food, while none of the control caches (set up by the researchers) were activated. The actual retrieval rate was probably higher, but the experiment faced some technical hurdles (4).

The ability to retrieve items from cache sites depends on spatial information such as visual cues. In (14), black-capped chickadees (Poecile atricapillus) were placed in an enclosure and their food-caching behavior was tracked. Object rearrangement around the enclosure greatly impaired the chickadees' ability to find their cache sites; manipulation of prominent 
birds' retrieval performance than small proximal objects (5-cm squares). Shifting objects by as little as $20 \mathrm{~cm}$ to the right significantly decreased the chickadees' ability to recover the food. Moreover, in almost $70 \%$ of the cases, the birds searched within $5 \mathrm{~cm}$ of the location implied by the more prominent landmarks, with a mean displacement of around $20 \mathrm{~cm}$. Assuming this is approximately the caching resolution, the finding indicates that a small area of 10x10 meters can hold as much as 2,500 potential caching sites.

The hippocampus plays a central part in the remarkable cognitive feat of caching (9). This is not surprising, as the hippocampus is known to be involved in processing spatial information in the brain (11). A large subpopulation of neurons within the hippocampus in animals such as mice, rats, and bats exhibit place-cell behavior; that is, they increase in their spike rate in response to the animal's entering a specific region within a given site (usually, but not always, one region per cell). The region activating each place cell often changes when the animal moves to a new area, often in an unpredictable manner. However, if the animal returns to a site previously visited, the place cell's receptive fields also return to their previous arrangement, and this change happens practically instantaneously. This remapping of the receptive field within a given environment is mostly insensitive to landmark manipulations. In primates, we usually find a related type of cells referred to as spatial view cells. These cells respond remotely when an animal is gazing at a specific region, independently of the animal's location or head direction (12).

A well-known homolog to the mammalian hippocampus also exists in birds, with similar involvement in spatial and episodic memory (13). Hippocampus size in birds was found to correlate with birds' ability to stash food. Although the interpretation of this correlation is under debate $(14,15)$, animals that used more cache sites generally had a larger hippocampus than non-caching bird species $(16,17)$. In addition, even within the same species, the size of the hippocampus was found to be larger in individuals dwelling in harsher environments that makes them more dependent of the cached food (18). Moreover, hippocampal neurogenesis has a seasonal element and seems to correlate with caching activity throughout the year (19). For many years, the spatially responsive cells found in avian brains were less related to a fixed position in space and more related to the challenge the animal faced such as the position of a goal within a maze (20). Only very recently the observations have led researchers to hypothesize that caching requires some form of spatial and episodic memory (22). 
Yet as birds and other animals need an internal mechanism to guide them to stash food in specific locations, the same mechanism can also be used to direct them to the exact same locations while retrieving the food in that area. Such a mechanism may serve as a mnemonic device (as suggested in (23)) or possibly replace the need for memory altogether. Such a pseudo-random approach is much simpler than remembering hundreds of thousands of stashing sites while still supporting all the existing empirical evidence. The guidance is based on prominent landmarks in the terrain, such as trees and rocks, which are not likely to substantially change over time, and can be used in the subsequent cache retrieval. And we already know of a specific set of neurons to be able to do precisely this - the previously mentioned hippocampal spatial cells. Spatial cells are unique to each individual, they assign scores and rankings (using spike rates) to different locations within each area, they persist over time, and remap when in the same area. We show how these properties allow animals to find their cache sites efficiently and secretly.

\section{Cryptography in the brain}

122 A mechanism, or mapping, that can facilitate efficient hiding and retrieval of multiple cache sites without relying on memory would need to have several basic properties. From a theoretical perspective, the class of methods that achieve this is known as cryptographichashing functions (or crypto-hashes, for short), which, as the name suggests, are comprised of two components: hashing functions and cryptographic keys (24).

In this context, hashing refers to a class of functions that map arbitrarily complex data (images, texts, audio files, and others) to a fixed size lower-dimensional representation. mapping them directly to a memory location (a type of a mnemonic device). In the case of food caching, hashing can be used to map a set of landmarks within and around an area onto a selected caching site within that area (Figure 1A). Efficient hash functions are such that the probability of assigning different cues the same output is kept to a minimum; This property reduces the possibility of collisions and redundancies that can occur when two different inputs result in the same output. It also makes better use of all the available resources - in the case of animals allowing the use of the entire area for caching.

Unlike standard hash functions, crypto-hashes incorporate an additional entity - a private key that renders the mapping unique to the key owner (24). Assuming no two individuals

141 have the same key, it also means that the mapping will result in unique caching sites within 142 the same area (Figure 1B). Another valuable property of crypto-hashes is that it is often 
difficult to infer the key from a small number of examples, so even if another animal finds several caches, it will not be able to deduce the location of all others.

A straightforward and biologically plausible realization of crypto-hashing is through a simple two-layer neural network model with sparse connectivity (Figure 1C; see supplementary information). The first layer, the input layer, represents the visual cues (landmarks) within a given patch of land (Figure 2A, 2B). The second layer is a 2D lattice of spatial neurons, in which each neuron points to a specific location in a given area (Figure 2C). The firing rate of each spatial neuron corresponds to the likelihood of choosing its particular location as a caching site. The spatial neurons are sparsely innervated by the input neurons.

In our model, we set the number of connections to a constant, typically equivalent to the number of landmarks the model uses (usually four). This sparse connectivity helps maintain a low number of potential cache sites by the output layer. A simple equation can summarize the activity of each spatial neuron

$$
C_{x_{i}, y_{i}}=\left|\sum_{x, y} w_{x, y}^{i} I_{x, y}\right|
$$

where $C_{x_{i}, y_{i}}$ is the score, or spike-rate, of the spatial neuron pointing to coordinate $x_{i}, y_{i}$ of the output grid; $I_{x, y}$ is the input from coordinates $x, y$; and $w_{x, y}^{i}$ represents the strength of the connectivity between $I_{x, y}$ and $C_{x_{i} y_{i}}$. The strength, or weight $w_{x, y}^{i}$, of the connections was assigned randomly at between one and minus-one. The inputs were also set in the range one and minus-one, where the absolute magnitude represents the landmark's prominence (one is very prominent and zero is designated as not noticeable). The sign represents the landmark type, for example positive values represent "trees", and negatives represent "rocks". The scores of the output neurons $C_{x_{i}, y_{i}}$ effectively determine the probability of their target area to be used as a cache site. We use the absolute value to keep the outputs positive, although it has no computational benefit to the model. Nor is the choice to distinguish between two types of objects by allowing negative inputs.

This neural network is a crypto-hash function, as it fulfills the three essential properties: (1) It maps a complex terrain into a point with minimal overlapping probability across the terrain or (2) across subjects (Figure 3A,3B), and (3) reconstructing or decrypting the mapping from examples is difficult. The third point stems from the fact that the connection to each output-layer neuron is chosen randomly and independently of the others. Thus it is effectively equivalent to holding a unique key for each neuron. 
A

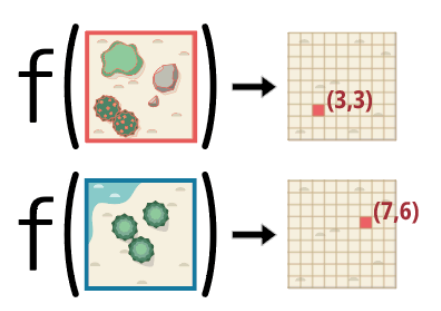

B

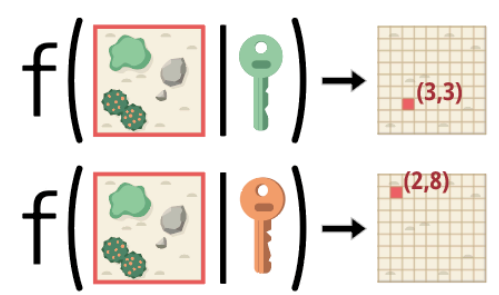

C
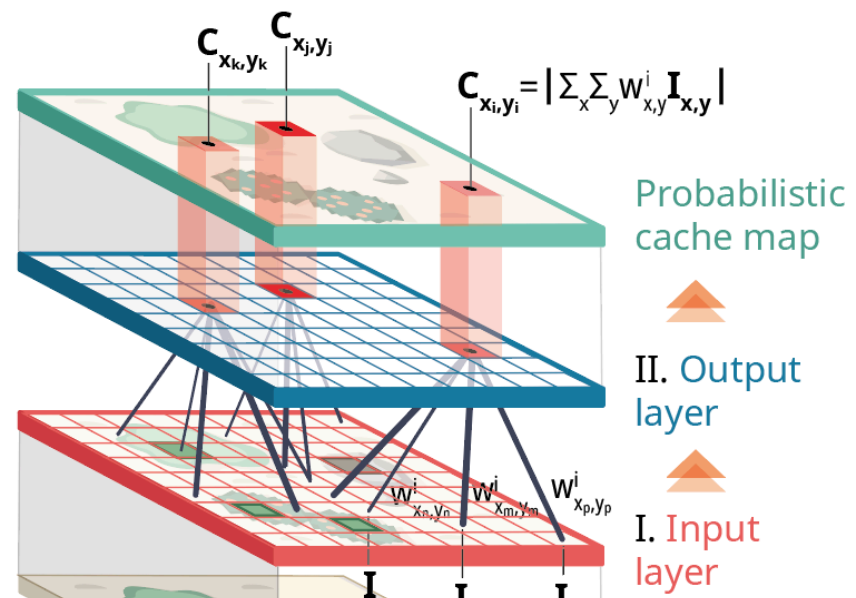

II. Output

layer

I. Input

layer

Terrain

Figure 1. Cryptographic mechanisms in the brain. (A) Hash functions take high-dimensional objects (a map of a complex terrain, for example) and map them into a low-dimensional representation such as a point within that terrain. (B) Crypto-hash functions also include a private key that makes mapping the same object unique across individuals with different keys. (c) Crypto-hashing in a two-layer neura/ network: Each neuron $\mathrm{I}_{\mathrm{x}, \mathrm{y}}$ in the input layer represents landmarks within a small square area. We assume two types of landmarks, which we refer to as 'trees' and 'rocks'. Neurons that point to trees are assigned a positive value, rocks get negative values, and if no object is within the neuron's receptive field, it is set to zero. The absolute value of $\mathrm{I}_{\mathrm{x}, \mathrm{y}}$ corresponds to an object's prominence; thus, prominent trees will get +1 , smaller trees -0.5 , and small rocks may be assigned a value of 0.3. The spatial output laver is a 20 mesh that assigns a caching score to each location within the site. The higher the score, the more likely this location would be used for caching. Each neuron in the output layer is innervated by a small number of input neurons. In all of our simulations, we matched this number to the number of landmarks the model uses. 


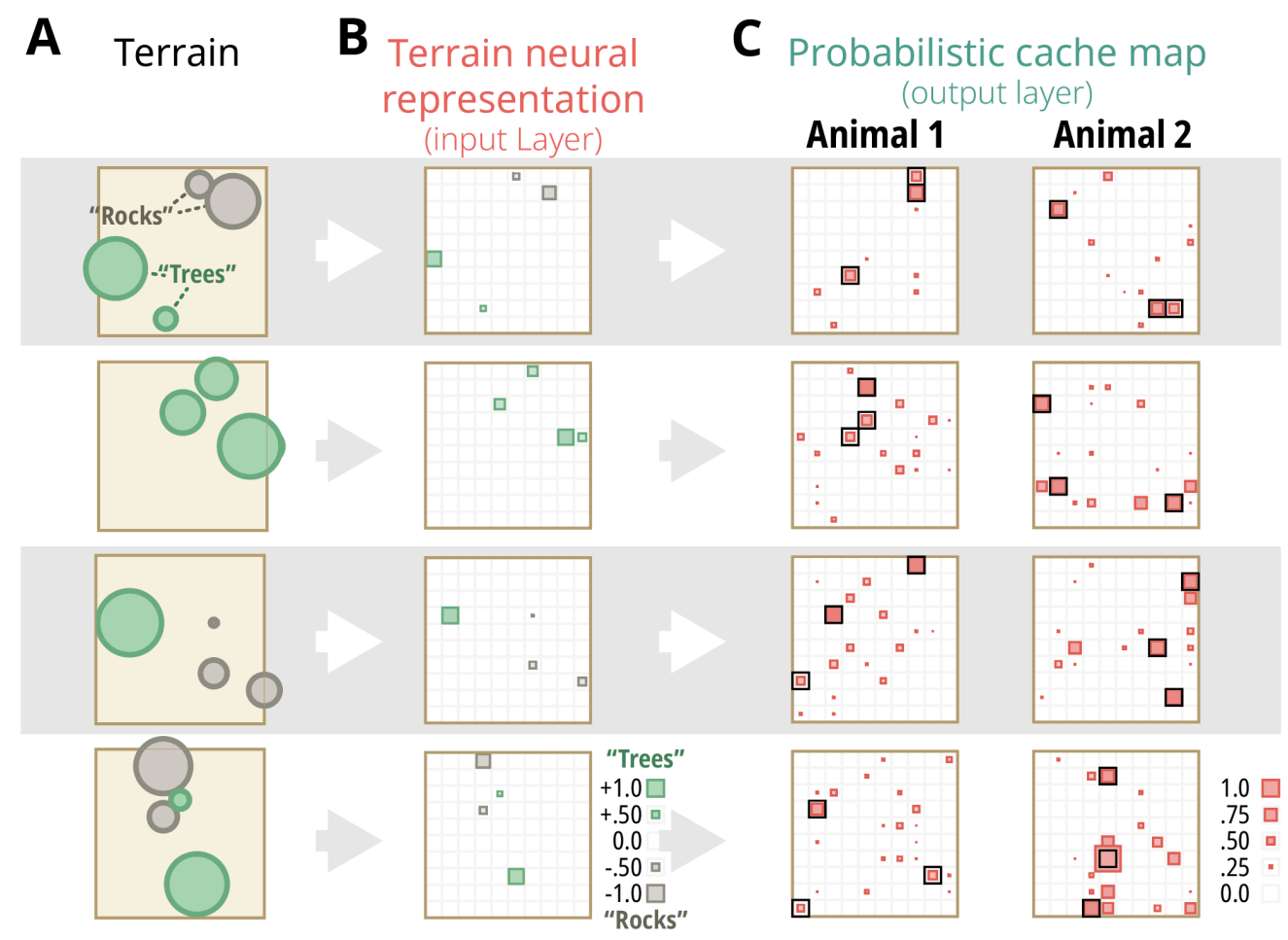

Figure 2. Using spatial features to map cache sites without requiring brain plasticity. (A-C) Four examples of the outcome of a crypto-hash neuronal network (in rows). (A) We simulated a terrain with four prominent landmarks by randomly choosing four cells within a $10 \times 10$ grid. The cells were assigned random values between -1 and +1 , so that the absolute value represents the prominence of the spatial feature (cells with values close to +1 and -1 being the most prominent), and the sign represents the type of object. We refer to positive-valued cells as "trees" and negative cells as "rocks" for brevity. (B) The representation of the landscapes from (A) in the neural network's input laver. The size of the colored inlaid boxes represents the object's prominence and their color its sign (green for positive values or "trees", and gray for negatives or "rocks"). (c) The output of the target layer of two randomly chosen neural networks (Animal 1 and 2 ) in response to the inputs in (B). The output laver creates a unique probabilistic map of possible cache sites.

180 The result can serve as a traditional crypto-hash function by choosing the target neuron with the highest score as the mapping outcome (see Materials an Methods). However, a probabilistic map with multiple outcomes of varying probabilities - apart from being more

183 biologically feasible - also provides several benefits. The most straightforward benefit is 184 that it allows for an arbitrary number of caching sites within each area by choosing the spatial neurons with the top scores.

187 Another valuable property of probabilistic maps is that they allow the addition of temporal 188 considerations into caching behavior (22). Assuming the order of food recovery starts with 
189 locations that have higher scores, items with higher nutritional values or perishable items 190 (such as dead insects, as opposed to seeds) could be stashed in places with higher scores 191 making them more likely to be recovered prior to items assigned to lower-scored locations 192 (Figure 3C). In addition, avoiding previously excavated sites requires only memorizing the 193 score of the last excavated and choosing only sites with a lower score (we refer to this 194 behavior as bookmarking).

195

196 Finally, we did not address how mappings in the model are kept allocentric and invariant to 197 the animal's position. A straightforward approach to achieve such invariance was 198 suggested in an elegant paper about geometric hashing (25). The method there is based on 199 choosing two prominent objects in the area and using them to scale and align all landmarks. 200 The vector connecting the two most prominent landmarks defines the direction axis and 201 the distance between them sets the scale unit. Using this approach, we can obtain a model 202 that is insensitive to affine transformations.
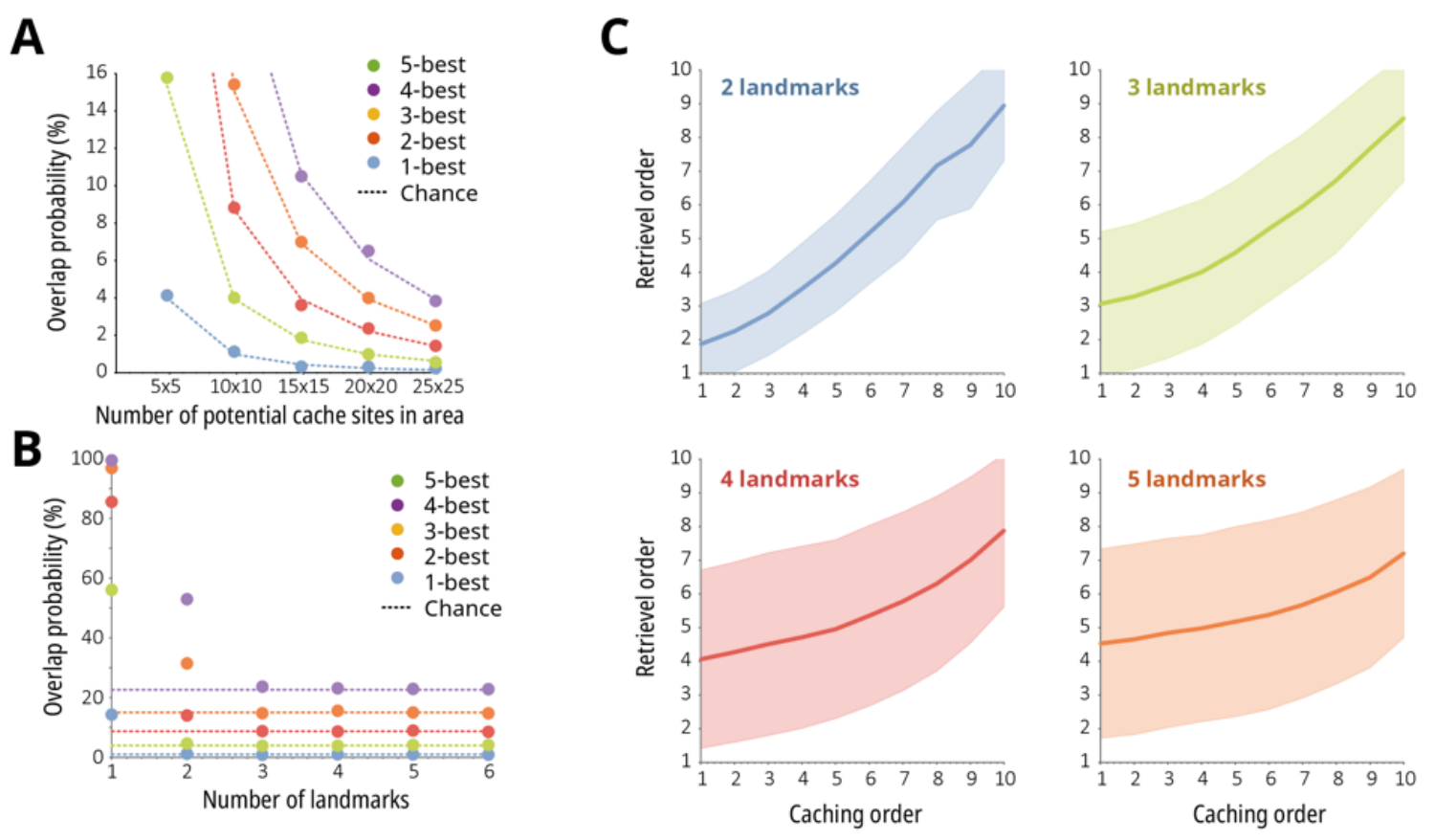

Figure 3. Properties of a crypto-hash neural network. The probability of overlapping sites between two random networks as a function of (A) potential cache sites and (B) the number of landmarks the network uses. The different colors correspond to the number of caches used in each area. The dotted lines are the overlap as expected from a random choice of sites. (c) The probabilistic nature of the target laver scores allows the network to maintain temporal dynamics. We assume that caching and retrieval order are determined by the target-laver score, from highest to lowest. If the choice is not absolute but probabilistic, we still get the same temporal dynamic in both phases. The shaded area around each line represents the standard deviation. 


\section{METHODS}

\section{Landmarks and terrain}

While the algorithm is not sensitive to the number of landmarks, for the sake of simplicity, we assumed a fixed number of landmarks within each area - four, in this paper. These landmarks were divided into two categories, which we refer to as "trees" and "rocks". The locations of the landmarks were chosen randomly and uniformly from an n-by-n square grid (in most cases we use $n=10$ ). Each landmark was then assigned a random value between minus one and one that signifies the objects category and prominence: Cells that have positive values are referred to as trees (values between zero and one), while rocks had negative numbers (between zero and minus one). The absolute value of each landmark signifies its prominence - so prominent trees have values closer to one and prominent rocks have values closer to minus one. Zero marks a no-object. The outcome is a sparse $n-b y-n$ matrix $S_{n \times n}$ with values that vary between minus one and one.

\section{Crypto-Hash Functions}

Hash functions map data with arbitrary dimensions to a fixed-length value (24). In mathematical terms, a hash function $g_{p}(\bar{s})$ is such that

$$
g_{p}(\bar{s})=\bar{y}_{p}
$$

where $\bar{s} \in S$ is a vector of arbitrary length, and $\bar{y}_{p}=\left(y_{1}, y_{2}, \ldots, y_{p}\right) \in \mathcal{Y}_{p}$ is a vector of a fixedlength $p$. Since the length of $\bar{s}$ is often larger than that of $\bar{y}_{p}$ hash-functions can be viewed as a special case of dimensionality-reduction.

An optimal hash function is such that the probability of mapping two inputs onto the same output is minimal, or, equivalently, that all outputs values should have (roughly) the same probability. This principal of uniformity can be formulated as

$$
p\left(g_{p}(\bar{s})=y\right) \approx \frac{1}{\left|\mathcal{Y}_{p}\right|}
$$

where $\left|\mathcal{Y}_{p}\right|$ is the cardinality (number of elements or size) of the set $\mathcal{Y}_{p}$ of all possible output values. Because of their uniformity, hash functions are often used in data storage and retrieval tasks as they allow data access at nearly a constant time while requiring a storage size that is only slightly larger than the space needed to store the data itself. 


$$
g_{p}(\bar{s} ; k)=\bar{y}_{p}
$$

243 The key ensures that the mapping is unique, i.e. the probability that the same inputs produce the same outputs for different keys is close to chance. Crypto-hash functions, like hash functions in general, are deterministic, meaning that the same combination of input and key will always produce the same output value. However, crypto-hash mappings are also one-way-functions, meaning that they are difficult to invert; knowing an output value gives very little information about the input or key.

\section{Crypto-Hash Neural Network}

251 Choice and retrieval of cache sites is based on prominent landmarks within a terrain. 252 Assuming $S_{n \times n}$ is the representation of the current area's terrain (see the 'simulated terrain' 253 section), our crypto-hash function can be defined as

$$
g_{p}\left(S_{n \times n} ; k\right)=(x, y)
$$

where $(x, y)$ is the cache coordinate within the area so that $x, y \in\{1, \ldots, n\}$, and $k$ is the crypto257 key.

A straightforward and biologically plausible to achieve this is using a neural network. We define a two-layer network where, for simplicity, the neurons on both layers are organized as a grid with $x, y$ indices. The value of each neuron in the first layer $I_{x, y}$, which is the input layer, is set according to the corresponding area tile or the $(x, y)^{\prime}$ th cell in $S_{n \times n}$. Each output layer neuron $C_{x_{i}, y_{i}}$ was sparsely connected to the input layer, and the weights $w_{x, y}^{i}$ were randomly distributed between minus-one and one. The value of output neurons is the

$$
C_{x_{i}, y_{i}}=\left|\sum_{x, y} w_{x, y}^{i} I_{x, y}\right| \text {. }
$$

In order to get a crypto-hash function in the form (1) we can take the index of the maximal 268 value or In our case, a unique key $k$ is obtained from the random choice of weights between the neural network's layers. 
273 However, as we mentioned in the text, keeping the function as a probabilistic mapping like

274 in (2) has several benefits and this is the form we use in the paper.

275

\section{DISCUSSION}

Although spatial cells, such as place cells have been extensively studied, the mechanism we presented is one of the few explanations to how the brain might utilize these cells. We proposed here that spatial cells can serve as the brain's crypto-hash functions, enabling animals to hide food in unique cache sites and later to retrieve it. So far, there has been no theory that explains what principles guide animals when choosing cache sites; If it was merely a question of optimality, all animals might end up choosing the same sites, which would lead to theft (or kleptoparasitism).

For brevity, we did not address how mappings in the model are kept allocentric and invariant to the animal's position. A straightforward approach to achieve such invariance was suggested in an elegant paper about geometric hashing (30). The method there is based on choosing the two most prominent objects in the area and using them to scale and align all landmarks. The vector connecting the two most prominent landmarks defines the direction axis and the distance between them sets the scale unit. Using this approach, we obtain a model that is insensitive to affine transformations (see supplementary information).

While our focus here is on scattered hoarding, a similar mechanism may also be involved in processes of decision making when the number of possibilities is large. A bee foraging for nectar and pollen, for example, would benefit from choosing flowers less likely to have been visited by other bees. The same function-driven mechanism (rather than memory-driven) may also help steer migratory animals back to previously used nesting areas. And since the hippocampus is involved in abstract knowledge in addition to spatial information (11), the scope of decision-making might be much broader; Since diversity is a key characteristic of all living system, it is tempting to think that humans' individualistic tendencies might also be somehow related to our proposed brain circuitry.

While the work we presented is theoretical, it raises some obvious predictions. First, that knowing the spiking patterns of spatial cells will enable us to determine cache site locations. Moreover, if we know the remapping between sites well, we can use it to decrypt the internal 
309 that the location of cache sites within a given area would be consistent across multiple hide

310 and retrieval iterations.

312 The instinct to choose cache sites that are both unique and obscure has a clear evolutionary 313 advantage. We therefore fondly suggest addressing this movement pattern as cryptotaxis 314 and the neurons involved as crypto-cells.

\section{Acknowledgments}

318 We would like to send our love and appreciation to Prof. David Sherry from Western 319 University for inspiring a large part of the idea for this paper with his beautiful works. We 320 thank Shiri Gerson for her help, wonderful comments, and lots of support. We also thank 321 Prof. Haim J. Wolfson for his help on the theory of hashing. Special thanks also to Prof. Anat 322 Barnea for her invaluable introduction to the world of scatter hoarding and the avian 323 hippocampus. We also thank Prof. Arnon Lotem for his insightful ideas, enthusiasm, and 324 encouragement.

\section{Funding}

327 This work was supported by the Israeli Science Foundation (ISF; Grant 2505/20) and the Ring 328 Center for Interdisciplinary Environmental Research.

\section{Competing interests}

331 Authors have no competing interests

332 Data and materials availability

333 All code is available online 


\section{Bibliography}

1. M. Stevens, S. Merilaita, Animal camouflage: current issues and new perspectives. Philosophical Transactions of the Royal Society B: Biological Sciences. 364, 423-427 (2009). ecology of crypsis, aposematism, and mimicry (Oxford University Press, 2019). 346202 (2009).

347 4. C. C. Smith, O. J. Reichman, The Evolution of Food Caching by Birds and Mammals. 348 Annual Review of Ecology and Systematics. 15, 329-351 (1984).

$3495 . \quad$ L. F. Jacobs, E. R. Liman, Grey squirrels remember the locations of buried nuts. Animal 350 Behaviour. 41, 103-110 (1991).

351 6. R. H. Yahner, The Adaptive Nature of the Social System and Behavior in the Eastern 352 Chipmunk, Tamias striatus. Behavioral Ecology and Sociobiology. 3, 397-427 (1978).

353 7. N. Tinbergen, Von den Vorratskammern des Rotfuchses: (Vulpes vulpes L.)1. 354 Zeitschrift für Tierpsychologie. 22, 119-149 (1965).

355 8. D. W. MacDonald, Food caching by red foxes and some other carnivores. Z 356 Tierpsychol. 42, 170-185 (1976).

357 9. A. Brodin, The history of scatter hoarding studies. Philos Trans R Soc Lond B Biol Sci. 358 365, 869-881 (2010).

359 10. T. A. Stevens, J. R. Krebs, Retrieval of stored seeds by Marsh Tits Parus palustris in the 360 field. Ibis. 128, 513-525 (1986).

361 11. T. E. J. Behrens, T. H. Muller, J. C. R. Whittington, S. Mark, A. B. Baram, K. L. 362 Stachenfeld, Z. Kurth-Nelson, What Is a Cognitive Map? Organizing Knowledge for Flexible 363 Behavior. Neuron. 100, 490-509 (2018).

364 12. E. T. Rolls, Spatial view cells and the representation of place in the primate 365 hippocampus. Hippocampus. 9, 467-480 (1999).

366 13. E. D. Jarvis, O. Güntürkün, L. Bruce, A. Csillag, H. Karten, W. Kuenzel, L. Medina, G. 367 Paxinos, D. J. Perkel, T. Shimizu, G. Striedter, J. M. Wild, G. F. Ball, J. Dugas-Ford, S. E. Durand, 368 G. E. Hough, S. Husband, L. Kubikova, D. W. Lee, C. V. Mello, A. Powers, C. Siang, T. V. 369 Smulders, K. Wada, S. A. White, K. Yamamoto, J. Yu, A. Reiner, A. B. Butler, Avian brains and 370 a new understanding of vertebrate brain evolution. Nature Reviews Neuroscience. 6, 151-159 371 (2005).

372 14. T. C. Roth, A. Brodin, T. V. Smulders, L. D. LaDage, V. V. Pravosudov, Is bigger always 373 better? A critical appraisal of the use of volumetric analysis in the study of the hippocampus. 374 Philos Trans R Soc Lond B Biol Sci. 365, 915-931 (2010).

375 15. D. F. Sherry, Neuroecology. Annu Rev Psychol. 57, 167-197 (2006). 
376 16. J. R. Krebs, D. F. Sherry, S. D. Healy, V. H. Perry, A. L. Vaccarino, Hippocampal 377 specialization of food-storing birds. Proc Natl Acad Sci U S A. 86, 1388-1392 (1989).

378 17. D. F. Sherry, A. L. Vaccarino, K. Buckenham, R. S. Herz, The hippocampal complex of 379 food-storing birds. Brain Behav Evol. 34, 308-317 (1989).

380 18. T. C. Roth, V. V. Pravosudov, Hippocampal volumes and neuron numbers increase 381 along a gradient of environmental harshness: a large-scale comparison. Proc Biol Sci. 276, 382 401-405 (2009).

383 19. A. Barnea, F. Nottebohm, Seasonal recruitment of hippocampal neurons in adult 384 free-ranging black-capped chickadees. Proc Natl Acad Sci U S A. 91, 11217-11221 (1994).

385 20. D. F. Sherry, S. L. Grella, M. F. Guigueno, D. J. White, D. F. Marrone, Are There Place 386 Cells in the Avian Hippocampus? BBE. 90, 73-80 (2017).

387 21. H. L. Payne, G. F. Lynch, D. Aronov, Precise spatial representations in the 388 hippocampus of a food-caching bird. bioRxiv (2020).

389 22. N. S. Clayton, A. Dickinson, Episodic-like memory during cache recovery by scrub jays. 390 Nature. 395, 272-274 (1998).

391 23. A. Barnea, F. Nottebohm, Patterns of food storing by black-capped chickadees 392 suggest a mnemonic hypothesis. Animal Behaviour. 49, 1161-1176 (1995).

393 24. C. Paar, J. Pelzl, Understanding Cryptography: A Textbook for Students and 394 Practitioners (Springer Science \& Business Media, 2009).

395 25. H. J. Wolfson, I. Rigoutsos, Geometric hashing: an overview. IEEE Comput. Sci. Eng. 4, 396 10-21 (1997). 